\title{
Electrostatic Free Energy and Other Properties of States Having Nonequilibrium Polarization. I*
}

\author{
R. A. Marcus \\ Department of Chemistry, Polylechnic Institule of Brooklyn, Brooklyn, New York
}

(Received July 11, 1955)

\begin{abstract}
Various processes such as electron transfer reactions, redox reactions at electrodes, and electronic excitation of dissolved ions may proceed by way of intermediate states whose electrical polarization is not in equilibrium with the field arising from the charges present. The usual expressions for the electrostatic free energy and for the differential equation satisfied by the potential assume that the polarization and the field are in equilibrium. Accordingly, these equations are of but limited applicability to these processes. In the present paper equations are derived for various properties of systems having such nonequilibrium electrostatic configurations. These properties include the free energy, energy, and entropy of the nonequilibrium system, and the spacial dependence of the electrostatic potential. The free energy, for example, will be used to calculate the probability of formation of nonequilibrium states in certain problems of physical interest.
\end{abstract}

\section{INTRODUCTION}

$I^{\mathrm{N}}$ recent years processes such as electron transfer reactions, redox reactions at electrodes, and electronic excitation of ions in the solid state and in solution have been the subject of considerable interest. These processes appear to involve intermediate states having nonequilibrium electrostatic configurations in the sense that the electrical polarization may not be that which would be predicted in the usual manner from the known charge distribution. For example, when a dissolved ion absorbs light, the electron is raised to an excited state almost instantaneously. A considerably longer time is required for the solvent's dipoles to readjust themselves to this new configuration. Thus, for some brief time after the absorption act, the electrical polarization of the system will not be in equilibrium with the charge distribution. This concept served $^{1}$ as a basis of a theory of the absorption spectrum of various halide ions in solution. In a different fashion, related considerations may apply to the other processes mentioned.

The results obtained in the present paper for the free energy and for other properties will later be applied to develop a quantitative theory of certain oxidationreduction reactions. A knowledge of the free energy of nonequilibrium states is of use in calculating the probability of their formation, as intermediates, in certain cases. The usual expressions given for the electrostatic free energy and energy cannot be used for this purpose. Similarly Poisson's equation for the electrostatic potential is not applicable in its commonly written form. Each of these equations assumed in their derivation that the electrical polarization at each point in the system was in equilibrium with the electric field there.

In the present paper, equations for these properties

* This research was supported by the Office of Naval Research under Contract No. Nonr $839(09)$. Reproduction in whole or in part is permitted for any purpose of the United States Government.

I R. Platzman and J. Franck, L. Farkas Memorial Volume (Oth Cooperative Printing Press, Haifa, 1952). of nonequilibrium states are derived. However, the treatment of systems containing electrodes will be reserved for a later communication. Such systems involve certain additional complexities arising from the presence of charges induced in the electrodes by the medium itself.

\section{THEORETICAL}

The electrical field in an equilibrium system is generally described in terms of two vectors, the electric field strength $\mathbf{E}$ and the dielectric displacement. The first is the negative gradient of the electrostatic potential and the second is defined in terms of the charge distribution, and is expressible in terms of $\mathbf{E}$ by an equation involving the dielectric constant of the medium. In contrast, it is the state of polarization of the medium rather than the dielectric constant itself which defines the behavior of a nonequilibrium state. Accordingly, the dielectric displacement vector is not useful in describing such states and will not be used here. However, the electrostatic potential and its negative gradient, $\mathbf{E}$, retain their significance and usefulness.

Three vectors instead of two will be used to describe a nonequilibrium state, the additional vector being required since these states have an extra degree of freedom (the polarization of the medium is no longer that dictated by the local value of the electric field strength). The three vectors chosen for this purpose will be the electric field strength, the polarization, and a quantity, $\mathbf{E}_{c}$, the electric field strength which the charge distribution would exert if it were in a vacuum rather than in a polarized medium [see Eq. (19)]. We proceed now to define the state of polarization of a nonequilibrium state more fully.

\section{$U$ - and $E$-Type Polarization}

In general the electrical polarization is considered to be the sum of electronic, atomic, and orientation contributions. The time required for each of these to adjust itself to some rapid change in the charge distribution is the order of $10^{-15}, 10^{-13}$, and $10^{-11}$ sec, respectively. 
Thus, in a very brief instant after a change in the charge distribution only the electronic polarization will adjust itself to the change in charges, while the other contributions will be those characteristic of the original charge distribution. A nonequilibrium situation will therefore temporarily prevail. Various other physical situations, such as certain types of fluctuations of the electrical polarization of a medium, can be imagined, in which one or more contributions to the polarization are in equilibrium with the charge distribution while the remaining contributions are not. These fluctuations will be discussed in greater detail in a paper on the theory of electron transfer reactions.

In the present paper the electrostatic free energy will be calculated for those nonequilibrium states whose polarization consists of two types, which will be termed the $U$-type and the $E$-type:

(i) The $U$-type polarization is not in equilibrium with the given charge distribution and is quite independent of it. The contribution of this type to the total electrical polarization at a point in the system will be denoted by a vector, $\mathbf{P}_{u}(\mathbf{r})$, defining the magnitude and direction of this polarization. The coordinates of the point are designated by the vector $\mathbf{r}$ drawn from any arbitrary origin to the point.

(ii) The $\mathbf{E}$-type polarization, denoted by $\mathbf{P}_{e}(\mathbf{r})$, is in equilibrium with the electric field arising from the charge distribution and from the $U$-type polarization. That is, the polarization $\mathbf{P}_{e}(\mathbf{r})$ can be calculated from the electric field strength at the point $\mathbf{r}$ in the usual way.

The total polarization $\mathbf{P}(\mathbf{r})$ at the point $\mathbf{r}$ is the vector sum of these.

$$
\mathbf{P}(\mathbf{r})=\mathbf{P}_{u}(\mathbf{r})+\mathbf{P}_{\epsilon}(\mathbf{r})
$$

In most nonequilibrium systems of physical interest, the E-type polarization will be electronic and the $U$-type, atomic and orientation polarization. However, the following treatment is quite general in this respect, and is applicable regardless of which contributions make up each type of polarization.

\section{The Charging Process}

The free energy of a nonequilibrium state of the system can be calculated if a reversible path for reaching for reaching that state can be found. The electrostatic free energy is generally defined as the reversible work done along this path. It is the difference in the electrostatic free energy of two states which is the property used in problems of physical interest, regardless of whether these states are equilibrium or nonequilibrium in nature. For example, the difference in free energy arising from electrostatic interactions, between any two states which do not differ in the number of each species of charged particles present, is given by the difference in work done in charging up each of the states. Accordingly, in any state the contribution to the free energy arising from the electrostatic interaction of charges with each other and with the polarized medium may be calculated by subtracting from the electrostatic free energy, $F$, the work, $W_{\text {iso, }}$, required to charge up the state in a vacuum when the charges are infinitely distant from each other.

The reversible charging process employed to create the type of nonequilibrium state described earlier will be performed in two stages:

(i) In the first stage the final value of the $U$-type polarization, $\mathbf{P}_{u}{ }^{0}(\mathbf{r})$ say, will be produced in a reversible manner by the formation of some appropriate charge distribution. Meanwhile the $E$-type polarization assumes some value, $\mathbf{P}_{e}{ }^{0}$ say.

(ii) In the second stage the polarization $\mathbf{P}_{u}{ }^{0}(\mathbf{r})$ will be held fixed but the charge distribution will be reversibly altered until the final charge distribution is obtained. During this process the E-type polarization changes from $\mathbf{P}_{e}{ }^{o}(\mathbf{r})$ to some function, $\mathbf{P}_{e}(\mathbf{r})$ say, whose value will be dictated by the charge distribution and by $\mathbf{P}_{u}{ }^{0}(\mathbf{r})$.

In this manner a system has been produced having the polarization, $\mathbf{P}_{e}(\mathbf{r})+\mathbf{P}_{u}{ }^{0}(\mathbf{r})$, where $\mathbf{P}_{u}{ }^{0}(\mathbf{r})$ is not, but $\mathbf{P}_{e}(\mathbf{r})$ is, in equilibrium with the field.

The reversible work performed in this charging process can be calculated if the potential $\psi(\mathbf{r})$ is known during each stage at each point of the system. Accordingly, an expression for $\psi(\mathbf{r})$ will first be given.

\section{Equation for the Electrostatic Potential}

A polarized volume element $d V$, having a polarization $\mathbf{P}$, exerts the same potential ${ }^{2}$ as does a dipole of moment $\mathbf{P} d V$. This is true regardless of whether the polarization has a value dictated by the local electric field, or whether it is quite independent of it. That is, this equivalence of polarization and dipole moment is applicable regardless of whether or not the system is an equilibrium one electrostatically, in the sense defined earlier.

The potential of a system consisting of a collection of charges and of dipoles can be written as the sum of contributions from each of these. A system consisting of charges and polarized volume elements can, therefore, be treated in a similar manner. Consequently, an expression for the electrostatic potential which treats a system in this way can be used for the present nonequilibrium case. Such an expression for the potential ${ }^{3} \psi\left(\mathbf{r}^{\prime}\right)$ at a point $\mathbf{r}^{\prime}$ of the system is given by Eq. (2).

$$
\begin{aligned}
& \psi\left(\mathbf{r}^{\prime}\right)=\int \frac{\rho(\mathbf{r}) d V}{\left|\mathbf{r}-\mathbf{r}^{\prime}\right|}+\int \frac{\sigma(\mathbf{r}) d S}{\left|\mathbf{r}-\mathbf{r}^{\prime}\right|} \\
& \quad+\int \mathbf{P}(\mathbf{r}) \cdot \nabla_{r} \frac{1}{\left|\mathbf{r}-\mathbf{r}^{\prime}\right|} d V,
\end{aligned}
$$

"See G. Joos, Theoretical Physics (Blackie and Son, Ltd., London, 1934), p. 267.

M. Mason and W. Weaver, The Electromagnetic Field (University of Chicago Press, Chicago, 1929), p. 67; compare reference 2 . 
where $\rho(\mathbf{r})$ is the charge density per unit volume at the point $\mathbf{r}$ of the system and $\sigma(\mathbf{r})$ is the charge density per unit area at a surface element $d S$ of an interface of the system. The surface integral is over all interfaces in the system. The polarization $\mathbf{P}$ is sum of the $E$ - and $U$-types and is given by Eq. (1). The subscript $r$ of the gradient operator $\nabla_{r}$ indicates that the differentiation involved in $\nabla$ is with respect to the coordinates of $\mathbf{r}$, and not of $\mathbf{r}^{\prime}$. In later expressions where this subscript is omitted it will nevertheless be tacitly implied.

\section{The Electrostatic Free Energy}

Equation (2) for the potential will now be applied to calculate the work done in each step of the two-stage charging process.

\section{(a) Stage I}

The $U$-type polarization $\mathbf{P}_{u}{ }^{0}(\mathbf{r})$ can be induced by charging up the system reversibly, such that the end of this stage of the charging process the charge density is $\rho^{0}(\mathbf{r})$ and surface charge density is $\sigma^{0}(\mathbf{r})$, say. Expressions are given later for determining the $\rho^{0}$ and $\sigma^{0}$ needed to produce any specified polarization function, $\mathbf{P}_{u}{ }^{0}(\mathbf{r})$.

When, during the course of this charging process, the charges have a fraction $\lambda$ of their final charge at the end of this stage, the corresponding charge distributions will be denoted by $\rho^{\lambda}(\mathbf{r})$ and $\sigma^{\lambda}(\mathbf{r})$. That is,

$$
\rho^{\lambda}=\lambda \rho^{0} \quad \text { and } \quad \sigma^{\lambda}=\lambda \sigma^{0} .
$$

Similarly $\psi^{\lambda}(\mathbf{r})$ and $\psi^{0}(\mathbf{r})$ will denote the potential at the stage $\lambda$ and at the end of Stage $I$ of this process, respectively. If no electrical saturation occurs, ${ }^{4}$ that is if at equilibrium each type of polarization is proportional to the local value of the electric field strength, then it can readily be shown that

$$
\psi^{\lambda}=\lambda \psi^{0} \text {. }
$$

To establish this equation the equilibrium relationships between $\mathbf{P}_{e}, \mathbf{P}_{u}$ and $\mathbf{E}$, which prevail during Stage I, will first be defined. If $\mathbf{E}^{\lambda}$ denotes the electric field strength, $-\nabla \psi^{\lambda}$, and if $\alpha_{e}$ and $\alpha_{u}$ denote the polarizabilities associated with each type of polarization, then ${ }^{4}$

$$
\mathbf{P}_{e}^{\lambda}=\alpha_{e} \mathbf{E}^{\lambda}, \quad \mathbf{P}_{u}^{\lambda}=\alpha_{u} \mathbf{E}^{\lambda} .
$$

The same relation between $\mathbf{P}_{e}$ and $\mathbf{E}$ will prevail in Stage II, but not that between $\mathbf{P}_{u}$ and $\mathbf{E}$.

It follows from Eqs. (1), (2), (3), and (5) that the potential $\psi^{\lambda}$ is given by Eq. (6).

\footnotetext{
Electrical saturation will be assumed to be absent, in the interest of simplicity. However, the two-stage charging process used here could presumably be applied to treat this more complicated case. $A$ modification which treats one system having local regions of saturated dielectric is described in a forthcoming paper on the theory of election transfer reactions.
}

$$
\begin{aligned}
& \psi^{\lambda}=\int \frac{\lambda \rho^{0} d V}{\left|\mathbf{r}-\mathbf{r}^{\prime}\right|}+\int \frac{\lambda \sigma^{0} d S}{\left|\mathbf{r}-\mathbf{r}^{\prime}\right|} \\
&-\int\left(\alpha_{e}+\alpha_{u}\right) \nabla \psi^{\lambda} \cdot \nabla \frac{1}{\left|\mathbf{r}-\mathbf{r}^{\prime}\right|} d V .
\end{aligned}
$$

Dividing by $\lambda$ we have

$$
\begin{aligned}
\frac{\psi^{\lambda}}{\lambda}=\int \frac{\rho^{0} d V}{\left|\mathbf{r}-\mathbf{r}^{\prime}\right|} & +\int \frac{\sigma^{0} d S}{\left|\mathbf{r}-\mathbf{r}^{\prime}\right|} \\
& -\int\left(\alpha_{e}+\alpha_{u}\right) \nabla\left(\frac{\psi^{\lambda}}{\lambda}\right) \cdot \nabla \frac{1}{\left|\mathbf{r}-\mathbf{r}^{\prime}\right|} d V .
\end{aligned}
$$

At the end of Stage I of the process, $\lambda=1$ and $\psi=\psi^{0}$. Thus, when $\lambda$ equals 1, Eq. (7) becomes

$$
\begin{aligned}
\psi^{0}(\mathbf{r})=\int \frac{\rho^{0} d V}{\left|\mathbf{r}-\mathbf{r}^{\prime}\right|}+ & \int \frac{\sigma^{0} d S}{\left|\mathbf{r}-\mathbf{r}^{\prime}\right|} \\
& -\int\left(\alpha_{\boldsymbol{e}}+\boldsymbol{\alpha}_{u}\right) \nabla \psi^{0} \cdot \nabla \frac{1}{\left|\mathbf{r}-\mathbf{r}^{\prime}\right|} d V .
\end{aligned}
$$

Now the electrostatic potential is a unique ${ }^{5}$ function of position. However, both $\psi^{\lambda} / \lambda$ and $\psi^{0}$ are seen from Eqs. (7) and (8) to be that potential which would be produced by the charge distribution, $\rho^{0}$ and $\sigma^{0}$, in a system whose polarizability in the volume element $d V$ is $\left(\alpha_{e}+\alpha_{u}\right)$. According to the uniqueness theorem, ${ }^{5}$ $\psi^{\lambda} / \lambda$ and $\psi^{0}$ must be equal. Equation (4) has therefore been established.

The work done in changing the charge density of a volume element $d V$ by an amount $d \rho^{\lambda}$ is $\psi^{\lambda} d \rho^{\lambda} d V$, and in changing the surface charge density of an area element $d S$ by an amount $d \sigma^{\lambda}$ is $\psi^{\lambda} d \sigma^{\lambda} d S$. The total work $W$ done in either stage of the two-stage charging process may be found by integrating this over the entire system and over the change in the charges in that stage. Accordingly, $W$ is given by Eq. (9).

$$
W=\int_{\lambda} \int_{V} \psi^{\lambda} \frac{d \rho^{\lambda}}{d \lambda} d \lambda d V+\int_{\lambda} \int_{S} \psi^{\lambda} \frac{d \sigma^{\lambda}}{d \lambda} d \lambda d S
$$

Introducing into this equation, Eqs. (4) and (3) for $\psi^{\lambda}, \rho^{\lambda}$, and $\sigma^{\lambda}$, and integrating it from $\lambda=0$ to $\lambda=1$, we obtain for the work done $W_{I}$ in the first stage of the charging process:

$$
W_{I}=\frac{1}{2} \int \psi^{0} \rho^{0} d V+\frac{1}{2} \int \psi^{0} \sigma^{0} d S
$$

\section{(b) Stage II}

In this stage the charge distribution is changed from its value at the end of Stage $I, \rho^{0}$ and $\sigma^{0}$, to the value at the end of Stage II, which will be denoted by $\rho(\mathbf{r})$ and

\footnotetext{
${ }^{5}$ Compare reference 3 , pp. $100,146$.
} 
$\sigma(\mathbf{r})$, say. At any stage $\lambda$ of this half of the charging process, the charge distribution will be designated as $\rho^{\lambda}(r)$ and $\sigma^{\lambda}(r)$, given by Eq. (11).

$$
\begin{aligned}
& \rho^{\lambda}=\rho^{0}+\lambda\left(\rho-\rho^{0}\right), \\
& \sigma^{\lambda}=\sigma^{0}+\lambda\left(\sigma-\sigma^{0}\right) .
\end{aligned}
$$

The fraction $\lambda$ increases from 0 to 1 during Stage II. The $U$-type polarization is held fixed at $\mathbf{P}_{u}{ }^{0}(\mathbf{r})$ throughout Stage II. The E-type polarization $\mathbf{P}_{e}{ }^{\lambda}(\mathbf{r})$ at the stage $\lambda$ is related to the electric field strength $-\nabla \psi^{\lambda}$, as in Eq. (5).

Introducing these quantities into Eq. (2) for $\psi$ we obtain as the value of $\psi$ during Stage II

$$
\begin{aligned}
\psi^{\lambda}\left(\mathbf{r}^{\prime}\right)=\int \frac{\rho^{0}+\lambda\left(\rho-\rho^{0}\right)}{\left|\mathbf{r}-\mathbf{r}^{\prime}\right|} d V+\int \frac{\sigma^{0}+\lambda\left(\sigma-\sigma^{0}\right)}{\left|\mathbf{r}-\mathbf{r}^{\prime}\right|} d S \\
\quad+\int\left(\mathbf{P}_{u}^{0}-\alpha_{e} \nabla \psi^{\lambda}\right) \cdot \nabla \frac{1}{\left|\mathbf{r}-\mathbf{r}^{\prime}\right|} d V .
\end{aligned}
$$

This potential $\psi^{\lambda}$ can be shown to depend linearly on $\lambda$ in the following way: We subtract Eq. (8) from Eq. (12) and observe that $\mathbf{P}_{u}{ }^{0}$ equals $-\alpha_{u} \nabla \psi^{0}$. Dividing the resulting equation by $\lambda$ we obtain

$$
\begin{aligned}
\frac{\psi^{\lambda}-\psi^{0}}{\lambda}=\int \frac{\rho-\rho^{0}}{\left|\mathbf{r}-\mathbf{r}^{\prime}\right|} d V+\int \frac{\sigma-\sigma^{0}}{\left|\mathbf{r}-\mathbf{r}^{\prime}\right|} d S \\
\quad-\int \alpha_{e} \nabla\left(\frac{\psi^{\lambda}-\psi^{0}}{\lambda}\right) \cdot \nabla \frac{1}{\left|\mathbf{r}-\mathbf{r}^{\prime}\right|} d V .
\end{aligned}
$$

Setting $\lambda=1$ in this equation it follows that

$$
\begin{aligned}
\psi-\psi^{0}=\int \frac{\rho-\rho^{0}}{\left|\mathbf{r}-\mathbf{r}^{\prime}\right|} d V & +\int \frac{\sigma-\sigma^{0}}{\left|\mathbf{r}-\mathbf{r}^{\prime}\right|} d S \\
& -\int \alpha_{e} \nabla\left(\psi-\psi^{0}\right) \cdot \nabla \frac{1}{\left|\mathbf{r}-\mathbf{r}^{\prime}\right|} d V .
\end{aligned}
$$

It is seen from these equations that both $\left(\psi^{\lambda}-\psi^{0}\right) / \lambda$ and $\left(\psi-\psi^{0}\right)$ are the potential which would be produced by the charge distribution, $\left(\rho-\rho^{0}\right)$ and $\left(\sigma-\sigma^{0}\right)$, in a system whose polarizability in the volume element $d V$ is $\alpha_{\ell}$. According to the uniqueness theorem these potentials are therefore equal.

$$
\left(\psi^{\lambda}-\psi^{0}\right) / \lambda=\psi-\psi^{0} .
$$

Introducing Eqs. (11) and (15) into Eq. (9), integrating the resulting equation from $\lambda=0$ to $\lambda=1$, we obtain for the work done during Stage II, $W_{I I}$,

$$
\begin{aligned}
W_{I I}=\frac{1}{2} \int\left(\psi+\psi^{0}\right)\left(\rho-\rho^{0}\right) d V & \\
& +\frac{1}{2} \int\left(\psi+\psi^{0}\right)\left(\sigma-\sigma^{0}\right) d S .
\end{aligned}
$$

The electrostatic free energy $F$ is the total work done in the over-all charging process and is given by Eq. (17).

$$
\begin{aligned}
F & =W_{I}+W_{I I} \\
& =\frac{1}{2} \int\left(\psi \rho+\psi^{0} \rho-\psi \rho^{0}\right) d V+\frac{1}{2} \int\left(\psi \sigma+\psi^{0} \sigma-\psi \sigma^{0}\right) d S .
\end{aligned}
$$

This expression for $F$ can be converted into a more useful form, giving $F$ in terms of the properties of the final state of the system only, namely in terms of $\mathbf{P}_{u}{ }^{0}(\mathbf{r}), \mathbf{E}(\mathbf{r})$ and the field strength $\mathbf{E}_{c}(\mathbf{r})$ directly due to the charges. To do this we use an equation established in Appendix I

$$
\begin{aligned}
& \int \psi^{i} \rho^{i} d V+\int \psi^{i} \sigma^{i} d S \\
&=-\int\left(P_{u}{ }^{i}+P_{e}{ }^{i}-E_{c}{ }^{i} / 4 \pi\right) \cdot E_{c}{ }^{j} d V
\end{aligned}
$$

where $i$ denotes either the superscript ${ }^{0}$ or no superscript, and $j$ has a similar significance. The dot in the integrand indicates the dot product of the vectors. The quantity $-\mathbf{E}_{\boldsymbol{c}}{ }^{i}$ is the gradient of the potential directly resulting from the charges that is, it is the electric field strength which the charges would exert if they were in a vacuum rather than in a polarized medium:

$$
E_{c}^{j}(\mathbf{r})=-\nabla_{r}\left[\int \frac{\rho^{j}\left(\mathbf{r}^{\prime}\right) d V^{\prime}}{\left|\mathbf{r}-\mathbf{r}^{\prime}\right|}+\int \frac{\sigma^{j}\left(\mathbf{r}^{\prime}\right) d S^{\prime}}{\left|\mathbf{r}-\mathbf{r}^{\prime}\right|}\right],
$$

where the integration is, as indicated by $d V^{\prime}$ and $d S^{\prime}$, over the coordinates of $\mathbf{r}^{\prime}$ and not of r. From Eqs. (17) and (18) we obtain for $F$

$$
F=\frac{1}{2} \int\left\{\frac{\mathbf{E}_{c} \cdot \mathbf{E}_{c}}{4 \pi}-\mathbf{P} \cdot\left(\mathbf{E}_{c}-\mathbf{E}_{c}{ }^{0}\right)-\mathbf{P}^{0} \cdot \mathbf{E}_{c}\right\} d V,
$$

where $\mathbf{P}^{0}$ and $\mathbf{P}$ are given by

$$
\begin{aligned}
\mathbf{P}^{0} & =\mathbf{P}_{u}{ }^{0}+\alpha_{e} \mathbf{E}^{0}, \\
\mathbf{P} & =\mathbf{P}_{u}{ }^{0}+\alpha_{e} \mathbf{E} .
\end{aligned}
$$

Inasmuch as $\mathbf{E}_{c}{ }^{0}$ and $\mathbf{E}^{0}$ are not characteristic of the final state of the system, but rather of the state of the system at the end of the first half of the charging process, it is desirable that they be eliminated from Eq. (20). To do this the following equation, established in Appendix II, will be used.

$$
\int \mathbf{P}^{0} \cdot\left(\mathbf{E}-\mathbf{E}_{c}\right) d V=\int \mathbf{P} \cdot\left(\mathbf{E}^{0}-\mathbf{E}_{c}{ }^{0}\right) d V .
$$


Rearranging the terms of this equation we obtain

$$
\begin{aligned}
\int\left(\mathbf{P} \cdot \mathbf{E}_{c}{ }^{0}-\mathbf{P}^{0} \cdot \mathbf{E}_{c}\right) d V \\
=\int\left(\mathbf{P} \cdot \mathbf{E}^{0}-\mathbf{P}^{0} \cdot \mathbf{E}\right) d V \\
=\int\left\{\left(\mathbf{P}_{u}{ }^{0}+\alpha_{e} \mathbf{E}\right) \cdot \mathbf{E}^{0}-\left(\mathbf{P}_{u}{ }^{0}+\alpha_{e} \mathbf{E}^{0}\right) \cdot \mathbf{E}\right\} d V \\
=\int \mathbf{P}_{u}{ }^{0} \cdot\left(\mathbf{E}^{0}-\mathbf{E}\right) d V .
\end{aligned}
$$

Remembering that $\mathbf{E}^{0}=\mathbf{P}_{u}{ }^{0} / \alpha_{u}$, the desired equation for $F$ can be obtained from Eqs. (20) and (24):

$$
F=\frac{1}{2} \int\left\{\frac{\mathbf{E}_{c} \cdot \mathbf{E}_{c}}{4 \pi}-\mathbf{P} \cdot \mathbf{E}_{c}+\mathbf{P}_{u} \cdot\left(\frac{\mathbf{P}_{u}}{\alpha_{u}}-\mathbf{E}\right)\right\} d V,
$$

where $\mathbf{P}$ is given by Eq. (22) and the superscript ${ }^{0}$ has been dropped from $\mathbf{P}_{u}{ }^{0}$.

Thus, the electrostatic free energy of a nonequilibrium state having $U$-type and $E$-type polarization is given by the preceding equation. The expression is seen to depend on the known $\mathbf{P}_{u}(\mathbf{r})$ and $\mathbf{E}_{c}(\mathbf{r})$, and also upon $\mathbf{E}(\mathbf{r})$. The vector point function $\mathbf{E}_{c}(\mathbf{r})$ is calculated from the known charge distribution according to Eq. (19). The electrostatic potential, and hence its negative gradient $\mathbf{E}$, may be calculated with the aid of Eq. (2). To do this it is convenient to convert this integral equation for $\psi$ into a differential equation, and to specify the boundary conditions. This is done in a later section.

In each problem of physical interest the $U$-type polarization function $\mathbf{P}_{u}(\mathbf{r})$ must be computed by some method in order to calculate the electrostatic free energy according to Eq. (25). The method of computing $\mathbf{P}_{u}$ will generally depend in part upon the nature of the particular problem. In some cases, for example, it will be shown that $\mathbf{P}_{u}$ will appear as a solution of an equation arising from the maximization of the free energy expression subject to certain restraints. This subject will be discussed in detail in later papers which will apply Eq. (25) to various physical and chemical problems.

A more intuitive but less rigorous derivation of Eq. (25) for the electrostatic free energy is given in Appendix IV.

The derivation of Eq. (25) for the electrostatic free energy assumes that all contributions to the $U$-type polarization were originally in equilibrium with the same charge distribution. A less common case would be one where one contribution to the $U$-type polarization was originally in equilibrium with one charge distribution, while the other contribution (if any) to the $U$-type polarization was originally in equilibrium with a different distribution. This problem can readily be handled, a three-stage charging process being used instead of a two-stage process, but appears to be of less physical interest and will not be treated here.

In the application of Eq. (25) it is useful to express the polarizabilities, $\alpha_{e}$ and $\alpha_{u}$, in terms of dielectric constants. The polarization of an equilibrium system satisfies $^{2}$ Eq. (26).

$$
\mathbf{D}=\mathrm{E}+4 \pi \mathbf{P}
$$

where $\mathbf{D}$, the dielectric displacement, equals $D \mathbf{E}, D$ being the dielectric constant. It follows from this that $\mathbf{P}$ and $\mathbf{E}$ are related according to Eq. (27).

$$
{ }_{4 \pi} \mathbf{P}=(D-1) \mathbf{E} .
$$

Since $\mathbf{P}=\alpha \mathbf{E}$, it follows that

$$
4 \pi \alpha=D-1 .
$$

When the electric field is either steady or slowly varying, then $D$ becomes the static dielectric constant, $D_{s}$. Further, according to Eq. (5), under these conditions

$$
\mathbf{P}=\mathbf{P}_{u}+\mathbf{P}_{e}=\left(\alpha_{u}+\alpha_{e}\right) \mathbf{E}
$$

so that $\alpha$ of Eq. (28) becomes $\alpha_{u}+\alpha_{e}$. Thus we have

$$
4 \pi\left(\alpha_{u}+\alpha_{e}\right)=D_{s}-1 \text {. }
$$

If, instead, an electric field alternates with a very high frequency, only the electronic polarization can follow the electric field. In that case, $D$ of Eq. (28) becomes $D_{o p}$, the optical dielectric constant, i.e., the square of the refractive index in the visible region of the spectrum, say. Further, $\mathbf{P}=\mathbf{P}_{\boldsymbol{e}}=\boldsymbol{\alpha}_{e} \mathbf{E}$ then, so that $\alpha$ of Eq. (28) becomes $\alpha_{e}$. Thus we have

$$
4 \pi \alpha_{e}=D_{o p}-1 \text {. }
$$

On the other hand, when the $E$-type polarization is electronic plus atomic then $D_{o p}$ should be replaced by $D_{i r}$, the optical dielectric constant in the infrared region of the spectrum, and we obtain

$$
4 \pi \alpha_{e}=D_{i r}=1 . \dagger
$$

In the case of water, we have ${ }^{6}$ at $25^{\circ} \mathrm{C} D_{s}=78.5$, $D_{o p}=1.8$, and $D_{i r}=5.5$.

${ }^{6}$ Collie, Hasted, and Ritson, Proc. Phys. Soc. (London) 60, 145 (1948)

$\dagger$ Nole added in proof. - The charge distribution at the end of Stage I of the charging process, denoted by $\rho^{0}$ and $\sigma^{0}$, is a fictitious distribution used to produce the specified $U$-type polarization, $\mathbf{P}_{u}(\mathbf{r})$. By contrast, the charge distribution at the end of Stage II is the actual distribution of charges on the ions of the system. The complete charging process is performed at fixed configuration of these ions, though they may be uncharged in Stage I and charged up in Stage II. Thus the reversible work given by Eq. (25) was also performed at fixed configuration of these ions. There is therefore an additional free energy term which should be considered, namely the entropy term associated with the preliminary formation of this given uncharged configuration of the ions from a random configuration. Let $e_{i}, c_{i}(\mathbf{r})$, and $c_{i}{ }^{0}$ denote the charge, the concentration, and the average concentration of ions of the ith species which make up the continuous volume charge distribution $\rho(\mathbf{r}) ; \rho(\mathbf{r})$ equals $\Sigma_{i} e_{i} c_{i}(\mathbf{r})$. Then, this additional entropy term associated with the formation of the given configuration from a random one is the well-known excess entropy of mixing and is given by $\mathrm{Eq}$. (25a):

$$
-k \Sigma_{i} \int c_{i}\left(\ln c_{i}^{0}-\ln c_{i}^{0}\right) d V .
$$

The total electrostatic contribution to the free energy includes this term and $F$ given by $\mathbf{E q}$. (25). It is the sum of these two 


\section{Energy and Entropy of Nonequilibrium Systems}

The form of the expression for the electrostatic energy and entropy of these nonequilibrium states will depend upon the nature of the $U$-type and $E$-type polarization. In most problems of physical interest the $E$-type polarization will be electronic and the $U$-type, atomic plus orientation. In the interest of brevity, the energy and entropy expressions will be derived for this case alone. Other cases offer no difficulty, however. It may again be remarked that it is the difference in electrostatic energy and entropy of two states which is of physical interest.

When the $E$-type polarization is electronic, the $U$-type is atomic plus orientation polarization. Therefore, no rearrangement of atoms occurs during Stage II of the charging process since $\mathbf{P}_{u}$ is held fixed. Since the process is performed at constant temperature, no change in the momentum distribution of the nuclei occurs either. There will therefore be no entropy change in Stage II. The corresponding energy change is therefore equal to the free energy change, $W_{I I}$, and is given by Eq. (16).

In Stage I, the system is of the conventional type electrostatically, and the electrostatic energy and entropy can be calculated in the usual way. Since $W_{I}$ is the electrostatic free energy of an equilibrium system at the end of Stage I, the usual thermodynamic formulas can be applied, giving the energy and entropy in terms of derivatives of the free energy with respect to temperature. Accordingly, the electrostatic energy $U_{I}$ and entropy $S_{I}$ at the end of Stage I are given by Eq. (31).

$$
U_{I}=\left(\frac{\partial W_{I} / T}{\partial 1 / T}\right)_{\mathrm{E}_{c^{0}}}, \quad S_{I}=-\left(\frac{\partial W_{I}}{\partial T}\right)_{\mathbf{E}_{c^{0}}},
$$

where $\mathrm{E}_{c}{ }^{0}$ is held constant in the differentiation.

It is concluded therefore that the electrostatic energy $U$ and entropy $S$ are given by Eqs. (32) and (33), respectively.

$$
\begin{aligned}
& U=\left(\frac{\partial W_{I} / T}{\partial 1 / T}\right)_{\mathbf{E}_{c^{0}}}+W_{I I}=F-T\left(\frac{\partial W_{I}}{\partial T}\right)_{\mathrm{E}_{c}} \\
& S=-\left(\frac{\partial W_{I}}{\partial T}\right)_{\mathrm{E}_{c^{0}}},
\end{aligned}
$$

where $F$ is given by Eq. (25), $W_{I}$ is given by Eq. (10). terms, rather than $F$ alone, which is the electrostatic free energy, $F_{\text {s, say }}$

$$
F_{i}=F+k T \Sigma_{i} \int c_{i}\left(\ln c_{i}-\ln c_{i}{ }^{0}\right) d V,
$$

where $F$ is given by $\mathrm{Eq}$. (25). In a very dilute solution $c_{i}$ equals $c_{i_{0}}$ and $F_{\mathrm{e}}$ equals $F$.

Similarly the total electrostatic contribution to the entropy is the sum of the term in Eq. (25a) and of $S$ given by Eq. (33) in the following section. In Eq. (32a) therefore the energy remains unaffected.

The variation process performed in the subsequent section "The Equilibrium State" is performed at a given ionic configuration.
With the aid of Eq. (18) this equation for $W_{I}$ can be converted to Eq. (34).

$$
W_{I}=-\frac{1}{2} \int\left(P_{u}+\alpha_{\theta} \mathrm{E}^{0}-\mathbf{E}_{c}{ }^{0} / 4 \pi\right) \cdot \mathbf{E}_{c}{ }^{0} d V
$$

In calculating $U$ and $S$ from Eqs. (32) and (33), a knowledge of $\mathbf{E}_{c}{ }^{0}$ is necessary. If the charge distribution with which $\mathbf{P}_{u}$ was in equilibrium is given, then $\mathbf{E}_{c}{ }^{0}$ can be determined from Eq. (19). Otherwise it can be calculated from the known $\mathbf{P}_{u}(\mathbf{r})$ and the known $\alpha_{e}$ of each volume element. To do this the intensity of the field $\mathbf{E}^{0}(\mathbf{r})$ with which $\mathbf{P}_{u}$ would be in equilibrium is calculated from the relation $\mathbf{E}^{0}=\mathbf{P}_{u} / \boldsymbol{\alpha}_{u}$. Next, the volume charge density $\rho^{0}$ and the surface charge density $\sigma^{0}$ are calculated ${ }^{7}$ from this known $\mathbf{E}^{0}(\mathbf{r})$. The quantity $\mathbf{E}_{c}{ }^{0}$ can then be determined from $\rho^{0}$ and $\sigma^{0}$ using Eq. (19).

Of particular interest in the application of these expressions to certain problems is the calculation of the difference in energy of two states, which differ in their charge distributions but have the same $\mathbf{P}_{u}(\mathbf{r})$, the same polarizabilities, and the same physical boundaries. This energy difference will generally consist of two contributions: (1) a difference in internal electronic energy of the charged (ionic) particles and (2) a difference in the electrostatic energy of interaction of the charged particles with each other and with the medium. It is the second difference which will be calculated here. This difference is the difference in the value of $U_{r}$ in the two states, where $U_{r}$ is the electrostatic energy of each state, as defined in Eq. (32), relative to the value it would have if the charged particles of that state were isolated and in a vacuum. If $W_{\text {iso }}$ is the work required to charge up the state when it consists of charged particles in a vacuum, then $W_{\text {iso }}$ is temperature independent and the electrostatic energy of that state, $\left(\partial W_{\text {iso }} / T\right) /(\partial 1 / T)$ simply equals $W_{\text {iso }}$ itself. Thus the quantity $U_{r}$ is given, according to Eq. (32), by the relation

$$
U_{r}=U-W_{\mathrm{iso}}=F-T\left(\frac{\partial W_{I}}{\partial T}\right)_{\mathbf{E}_{c^{0}}}-W_{\mathrm{iso}}
$$

In charging up systems to produce each of the two polarized states by the charging process used here, it is seen that $W_{I}$ will be the same in each case, since $W_{I}$ depends only on $\mathbf{P}_{u}(\mathbf{r})$. Accordingly, the difference in the (relative) electrostatic energy $U_{r}$ of two states having the same $\mathbf{P}_{u}(\mathbf{r})$ but different charge distributions is given by $\mathbf{E q}$. (35).

$$
\Delta U_{r}=\Delta F-\Delta W_{\text {iso. }}
$$

\section{The Equilibrium State}

It is of interest to verify that $\mathbf{P}_{u}$ (r) assumes its equilibrium value, $\alpha_{u} \mathbf{E}(\mathbf{r})$, when the free energy expression

\footnotetext{
${ }^{7}$ The quantities $\rho^{0}, \sigma^{0}, \mathbf{E}^{0}$, and $\mathbf{P}_{u}$ are in "electrostatic equilibrium" with each other, so that the usual expressions for $\rho^{0}$ and $\sigma^{0}$ can be used. We have, for example, $\rho^{0}=\nabla \cdot \mathbf{E}^{0} / 4 \pi D=-\nabla^{2} \psi^{0} / 4 \pi D$. Similarly, $\sigma^{0}$ can be calculated from the surface divergence of $\mathbf{E}^{0}$ at the interface. Compare reference 2, p. 254.
} 
is minimized with respect to the vector point function $\mathbf{P}_{u}(\mathbf{r})$. A method which is somewhat similar to that described below will later be used in a theory of electron transfer reactions to determine the most probable configuration of various intermediate states.

Using Eq. (25), the variation $\delta F$ is first calculated and set equal to zero. The variation, performed at fixed charge distribution, i.e., at $\delta \mathbf{E}_{c}=0$, is given by

$$
\delta F=\frac{1}{2} \int\left(-\delta \mathbf{P} \cdot \mathbf{E}_{c}+\frac{2 \mathbf{P}_{u}}{\alpha_{u}} \cdot \delta \mathbf{P}_{u}-\mathbf{P}_{u} \cdot \delta \mathbf{E}-\delta \mathbf{P}_{u} \cdot \mathbf{E}\right) d V .
$$

Introducing the value of $\delta \mathbf{P}$ obtained from the variation of $\mathrm{Eq}$. (1),

we have

$$
\delta \mathbf{P}(\mathbf{r})=\delta \mathbf{P}_{u}(\mathbf{r})+\delta \mathbf{P}_{e}(\mathbf{r})=\delta \mathbf{P}_{u}+\alpha_{e} \delta \mathbf{E},
$$

$$
\begin{aligned}
\delta F=\frac{1}{2} \int\left\{\left(\frac{2 \mathbf{P}_{u}}{\alpha_{u}}-\mathbf{E}_{c}-\mathbf{E}\right) \cdot \delta \mathbf{P}_{u}\right. \\
\left.-\left(\alpha_{e} \mathbf{E}_{c}+\mathbf{P}_{u}\right) \cdot \delta \mathbf{E}\right\} d V=0 .
\end{aligned}
$$

However, $\delta \mathbf{P}_{u}$ and $\delta \mathbf{E}$ are not independent variations, and are related in the following way. In Appendix III it is shown that.

$$
\int \mathbf{P} \cdot \delta\left(\mathbf{E}-\mathbf{E}_{c}\right) d V=\int\left(\mathbf{E}-\mathbf{E}_{c}\right) \cdot \delta \mathbf{P} d V
$$

where $\delta \mathbf{E}_{c}=0$ in the present case. Introducing Eq. (36) for $\delta \mathbf{P}$ into this equation and rearranging terms we obtain as the relation between $\delta \mathbf{P}_{u}$ and $\delta \mathbf{E}$,

$$
\int\left(\alpha_{e} \mathbf{E}_{c}+\mathbf{P}_{u}\right) \cdot \delta \mathbf{E} d V=\int\left(\mathbf{E}-\mathbf{E}_{c}\right) \cdot \delta \mathbf{P}_{u} d V
$$

Using this equation, Eq. (37) for $\delta F$ becomes

$$
\delta F=\frac{1}{2} \int\left(\frac{2 \mathbf{P}_{u}}{\alpha_{u}}-2 \mathbf{E}\right) \cdot \delta \mathbf{P}_{u} d V=0 .
$$

Since this equation must hold for all possible variations of $\mathbf{P}_{u}$ in each volume element it follows that in each volume element the coefficient of $\delta \mathbf{P}_{u}$ is equal to zero. That is, at equilibrium we find

$$
\mathbf{P}_{u}=\alpha_{u} \mathbf{E} \text {. }
$$

\section{Field Equation and Boundary Conditions}

\section{(a) Field Equation}

Using Green's theorem, Eq. (2) for the electrostatic potential $\psi\left(\mathbf{r}^{\prime}\right)$ can be rewritten as ${ }^{8}$

$$
\psi\left(\mathbf{r}^{\prime}\right)=\int \frac{\rho-\nabla \cdot \mathbf{P}}{\left|\mathbf{r}-\mathbf{r}^{\prime}\right|} d V+\int \frac{\sigma+P_{n}}{\left|\mathbf{r}-\mathbf{r}^{\prime}\right|}-d S,
$$

where $P_{n}$ is the component of $\mathbf{P}$ which is normal to the surface element $d S$, the normal being directed from the medium to the surface.

\footnotetext{
${ }^{8}$ Compare reference 3, p. 57, Eq. (44).
}

A differential equation for the electrostatic potential $\psi\left(\mathbf{r}^{\prime}\right)$ may be obtained from Eq. (41) by operating on that equation with the operator $\nabla_{r^{\prime}}$. The subscript $r^{\prime}$ indicates that $\nabla_{r^{\prime}}$ involves differentiation with respect to the coordinates of $\mathbf{r}^{\prime}$. In this manner we obtain ${ }^{9}$

$$
\nabla^{2} \psi=-4 \pi(\rho-\nabla \cdot \mathbf{P})
$$

Introducing the relation $\mathbf{P}=\mathbf{P}_{u}-\alpha_{e} \nabla \psi$, we obtain the desired differential equation.

$$
\left(1+4 \pi \alpha_{\varepsilon}\right) \nabla^{2} \psi=-4 \pi\left(\rho-\nabla \cdot \mathbf{P}_{u}\right) .
$$

This equation reduces to Poisson's equation, $\nabla^{2} \psi$ $=-4 \pi \rho / D$, when $\mathbf{P}_{u}$ assumes its equilibrium value $-\alpha_{u} \nabla \psi$ (for $1+4 \pi \alpha_{e}+4 \pi \alpha_{u}$ equals $D$, according to Eq. (28a)).

\section{(b) Boundary Condition at an Inter face}

At any surface the boundary condition may be inferred from Eq. (41) by available methods. It is found to be ${ }^{10}$

$$
\frac{\partial \psi}{\partial n_{1}}+\frac{\partial \psi}{\partial n_{2}}=4 \pi\left(\sigma+P_{n_{1}}+P_{n_{2}}\right)
$$

where $\partial \psi / \partial n_{1}$ and $P_{n_{1}}$ are the normal components of $-\mathbf{E}$ and of $\mathbf{P}$, respectively, along a normal to the surface, the normal being directed from medium 1 to the surface. The corresponding quantities in medium 2 are designated by the subscript 2 .

This equation can now be expressed in terms of the normal component of $\mathbf{P}_{u}$ in media 1 and $2, P_{u, n_{1}}$ and $P_{u, n_{2}}$, say. We first observe that the relation between $P_{n_{1}}$ and $P_{u, n_{1}}$ is given by Eq. (45).

$$
\begin{aligned}
P_{n_{1}} & =P_{e, n_{1}}+P_{u_{i} n_{1}} \\
& =-\alpha_{e 1} \frac{\partial \psi}{\partial n_{1}}+P_{u, n_{1}}
\end{aligned}
$$

where $\alpha_{e_{1}}$ is the value of $\alpha_{e}$ in medium 1. An analogous equation exists for medium 2 . Introducing these equations into Eq. (44) we obtain the desired boundary condition.

$$
\begin{aligned}
\left(1+4 \pi \alpha_{\theta 1}\right) \frac{\partial \psi}{\partial n_{1}}+\left(1+4 \pi \alpha_{\theta 2}\right) & \frac{\partial \psi}{\partial n_{2}} \\
& =4 \pi\left(\sigma+P_{u, n_{1}}+P_{u, n_{2}}\right) .
\end{aligned}
$$

${ }^{\circ}$ Compare reference 3 , p. 93. In the expression given there, $\rho^{\prime}$ is to be replaced by $4 \pi(\rho-\nabla \cdot P), \eta^{\prime}$ by $4 \pi\left(\sigma+P_{n}\right)$, and $\mu^{\prime}$ is to be set equal to zero in order to obtain Eq. (42) of the present paper from $\mathrm{Eq} .(68)$ of that text, p. 96 , using the method outlined there for differentiating twice under the integral sign.

10 See reference 3, p. 97. To apply the method outlined there, we first note that the last term of Eq. (41) of the present paper is to be integrated over both sides of each surface. This term may, therefore, be rewritten as

$$
\int\left\{\left(\sigma+P_{n_{1}}+P_{n_{2}}\right) d S /\left|\mathbf{r}-\mathbf{r}^{\prime}\right|\right\}
$$

where the integration is only over one side of the surface, either side, and 1 and 2 refer to the two media which form the interface. Equation (44) of the present paper may then be obtained by replacing $\eta$ of Eq. (70), reference 3 , by $4 \pi\left(a+P_{n_{1}}+P_{n_{2}}\right)$. Their $\partial \Phi / \partial n_{1}$ is our $-\partial \psi / \partial n_{1}$. 
It is readily verified that this equation reduces to the customary equilibrium boundary condition when $\mathbf{P}_{u}$ assumes its equilibrium value.

\section{(c) Boundary Condition for an Ion}

The boundary conditions and approximations commonly used in equilibrium systems containing ions will first be discussed, and illustrated with a derivation of the free energy expression for an isolated ion and for interacting ions. Analogous conditions and approximations will then be derived for nonequilibrium conditions.

Ions are usually treated as spherical conductors. The boundary condition at the surface of a conductor and, therefore, at the surface of each ion is, for an equilibrium system:I1 $\psi$ is continuous at this surface, and further

$$
\int D \frac{\partial \psi}{\partial n} d S=4 \pi e
$$

and

$$
\psi=\text { constant in each ion, }
$$

where $e$ is the ionic charge and $\partial \psi / \partial n$ is the component of the electric field strength, $\mathbf{E}$, along a normal to the surface, directed from the dielectric medium to the surface of the ion.

When the system contains an isolated ion in a dielectric medium, it is readily verified that the expression for $\psi$ which satisfies the appropriate differential equation in the dielectric $\left(\nabla^{2} \psi=0\right)$, and which satisfies the above boundary conditions and the boundary condition that $\psi$ is zero at infinity, is given by Eq. (49).

$$
\left.\begin{array}{ll}
\psi(\mathbf{r})=e / D r, & r \geq a \\
\psi(\mathbf{r})=e / D a, & r \leq a
\end{array}\right\}
$$

where $a$ is the ionic radius. Calculating $\mathbf{E}$, the negative gradient of $\psi$, from this equation, the electrostatic free energy for this ionic system can then be shown to be given by Eq. (51), using Eq. (50) for the free energy of an equilibrium system.

$$
\begin{aligned}
& F=\int \frac{\mathbf{E} \cdot \mathbf{E} d V}{8 \pi D}, \\
& F=e^{2} / 2 a D .
\end{aligned}
$$

The usual Born ${ }^{12}$ expression for the free energy of solvation of an ion is then obtained by subtracting from this the value of $F$ in a vacuum, which is $e^{2} / 2 a$ according to Eq. (51) since the dielectric constant of a vacuum is unity.

In the case of two ions which are separated by a distance, $R$, the usual equation used for the free energy is

$$
F=\frac{e_{1}^{2}}{2 D a_{1}}+\frac{e_{2}^{2}}{2 D a_{2}}+\frac{e_{1} e_{2}}{D R}
$$

${ }^{11}$ See reference 3, p. 146. Their $\partial \Phi / \partial n$ is our $-\partial \psi / \partial n$.

12 M. Born, Z. Physik 1, 45 (1920). where $e_{1}$ and $e_{2}$ are the ionic charges, and $a_{1}$ and $a_{2}$ are the ionic radii. The first two terms are the solvation terms of the isolated ions [compare Eq. (51)], while the third term is, of course, the Coulombic free energy of interaction of the ions. An expression for the electric field strength $E$ which yields Eq. (52) when introduced into $\mathrm{Eq}$. (50) is $\mathbf{E}=-\nabla \psi$, where $\psi$ is given by

$$
\begin{aligned}
& \psi=\frac{e_{1}}{D r_{1}}+\frac{e_{2}}{D r_{2}} \quad r_{1} \geq a_{1}, \quad r_{2} \geq a_{2} \\
& \psi \cong \text { constant } \quad r_{1}<a_{1} \quad \text { or } \quad r_{2}<a_{2},
\end{aligned}
$$

where $\psi$ is the potential at a point distant $r_{1}$ and $r_{2}$ from the centers of the ions. While this solution for $\psi$ does satisfy Laplace's equation, $\nabla^{2} \psi=0$, it will satisfy the boundary conditions discussed earlier only if the ions are essentially point charges. Clearly, for example, $\psi$ given by Eq. (53) has an essentially constant value on the surface of the ion only when the ions are either very small or far apart. In fact, only under these conditions are Eqs. (53) and (54) mutually consistent. The approximation discussed here may be termed the point charge approximation, or perhaps more appropriately, the pseudo point charge approximation, since finite radii of the ions are used in Eqs. (53) and (54), and in the integration of Eq. (50).

It is readily verified that Eqs. (53) and (54) for $\psi$ satisfy the boundary condition given by Eqs. (47) and (48), when the point charge approximation is made: $\partial \psi / \partial n$ is calculated from Eq. (53) and integrated over the surface of one of the ions, ion 1 say. The contribution of the second term in the right-hand side of Eq. (53) to this surface integral is found to be zero when the ion is essentially a point, while the contribution of the first term to the integral is found to be $4 \pi e_{1} / D$. Adding these it is seen that Eq. (47) is satisfied.

It follows from these considerations that the interaction free energy of two ions is $e_{1} e_{2} / D R$ only for point charges.

In the treatment of ions in nonequilibrium dielectric media the same type of boundary conditions will be introduced. Integrating Eq. (46) over the surface of the $i$ th ion, and observing that in a conductor $P u_{n}$ and $E_{n}$ vanish, we obtain as the desired boundary condition,

$$
\begin{aligned}
\int\left(1+4 \pi \alpha_{e}\right) \frac{\partial \psi}{\partial n} d S & =4 \pi\left(e_{1}+\int P_{u_{n}} d S\right) \\
\psi & =\text { constant in each ion }
\end{aligned}
$$

where $\alpha_{e}, \partial \psi / \partial n$, and $P_{u_{n}}$ refer to the values of these quantities on the dielectric side of the iondielectric interface. It can be verified by using Eq. (28a) that Eq. (55) reduces at once to Eq. (47), when $P_{u_{n}}$ assumes its equilibrium value, $-\alpha_{u} \partial \psi / \partial n$.

For two (or more) ions interacting in a medium, the point charge approximation is introduced by treating 
the surface of integration as a very small surface about the center of each ion, as in the equilibrium case. The values of $\partial \psi / \partial n$ and $P_{u_{n}}$ at the surface are, as before, those which the medium would have if it extended up to the small surface.

\section{APPENDIX I. PROOF OF THE VALIDITY OF EQ.}

It will first be assumed and later proved that

$$
\begin{aligned}
\int \frac{\rho(\mathbf{r})}{\left|\mathbf{r}-\mathbf{r}^{\prime}\right|} d V+\int \frac{\sigma(\mathbf{r})}{\left|\mathbf{r}-\mathbf{r}^{\prime}\right|} d S & \\
& =-\int \frac{\mathbf{E}_{c}(\mathbf{r})}{4 \pi} \cdot \nabla_{r} \frac{1}{\left|\mathbf{r}-\mathbf{r}^{\prime}\right|} d V .
\end{aligned}
$$

Equation (18) can then readily be established as follows: Using Eq. (56), Eq. (2) for the potential becomes,

$$
\psi\left(\mathbf{r}^{\prime}\right)=\int\left(\mathbf{P}-\mathbf{E}_{c} / 4 \pi\right) \cdot \nabla_{r} \frac{1}{\left|\mathbf{r}-\mathbf{r}^{\prime}\right|} d V .
$$

Thus, the left-hand side of Eq. (18) is, upon changing the variable there from $\mathbf{r}$ to $\mathbf{r}^{\prime}$ and introducing Eq. (57) for $\psi\left(\mathbf{r}^{\prime}\right)$,

$$
\begin{aligned}
\mathrm{lhs}= & \iint\left(\mathbf{P}^{i}-\mathbf{E}_{c}^{i} / 4 \pi\right) \cdot \nabla_{r} \frac{1}{\left|\mathbf{r}-\mathbf{r}^{\prime}\right|} d V \rho^{j}\left(\mathbf{r}^{\prime}\right) d V^{\prime} \\
& +\iint\left(\mathbf{P}^{i}-\mathbf{E}_{c}{ }^{i} / 4 \pi\right) \cdot \nabla_{r} \frac{1}{\left|\mathbf{r}-\mathbf{r}^{\prime}\right|} d V \sigma^{i}\left(\mathbf{r}^{\prime}\right) d S^{\prime} .
\end{aligned}
$$

This equation may then be written as

$$
\begin{aligned}
\mathrm{lhs}=\int\left(\mathbf{P}^{i}-\mathbf{E}_{c}^{i} / 4 \pi\right) \cdot \nabla_{r}\left\{\int \frac{\rho^{i}\left(\mathbf{r}^{\prime}\right)}{\left|\mathbf{r}-\mathbf{r}^{\prime}\right|} d V^{\prime}\right. \\
\\
\left.\quad+\int \frac{\sigma^{j}\left(\mathbf{r}^{\prime}\right)}{\left|\mathbf{r}-\mathbf{r}^{\prime}\right|} d S^{\prime}\right\} d V .
\end{aligned}
$$

It is seen from Eq. (19) for $\mathrm{E}_{c}{ }^{i}$ that $-\nabla_{r}$ operating on the term in brackets in Eq. (59) is simply $\mathbf{E}_{c}{ }^{j}$ so that Eq. (59) becomes

$$
\text { lhs }=-\int\left(\mathbf{P}^{i}-\mathbf{E}_{c}{ }^{i} / 4 \pi\right) \cdot \mathbf{E}_{c}{ }^{i} d V .
$$

This is simply the rhs of Eq. (18).

It now remains to establish the validity of Eq. (56) in order to complete the proof. Introducing the definition of $\mathbf{E}_{c}[\mathrm{Eq}$. (19)] into the rhs of Eq. (56) we obtain

$$
\begin{aligned}
\text { rhs of Eq. (56) } & =\frac{1}{4 \pi} \int \nabla_{r}\left\{\int \frac{\rho\left(\mathbf{r}^{\prime \prime}\right)}{\left|\mathbf{r}-\mathbf{r}^{\prime \prime}\right|} d V^{\prime \prime}\right. \\
& \left.+\int \frac{\sigma\left(\mathbf{r}^{\prime \prime}\right)}{\left|\mathbf{r}-\mathbf{r}^{\prime \prime}\right|} d S^{\prime \prime}\right\} \cdot \nabla_{r} \frac{1}{\left|\mathbf{r}-\mathbf{r}^{\prime}\right|} d V,
\end{aligned}
$$

where in Eq. (19) the integration variable $\mathbf{r}^{\prime \prime}$ has been used instead of $\mathbf{r}^{\prime}$. Interchanging the order of integration in Eq. (61) we obtain

$$
\begin{aligned}
& \text { rhs of Eq. }(56)=\int \rho\left(\mathbf{r}^{\prime \prime}\right) I\left(\mathbf{r}^{\prime \prime}, \mathbf{r}^{\prime}\right) d V^{\prime \prime} \\
& +\int \sigma\left(\mathbf{r}^{\prime \prime}\right) I\left(\mathbf{r}^{\prime \prime}, \mathbf{r}^{\prime}\right) d S^{\prime \prime}
\end{aligned}
$$

where

$$
I\left(\mathbf{r}^{\prime \prime}, \mathbf{r}^{\prime}\right)=\frac{1}{4 \pi} \int \nabla_{r} \frac{1}{\left|\mathbf{r}-\mathbf{r}^{\prime \prime}\right|} \cdot \nabla_{r} \frac{1}{\left|\mathbf{r}-\mathbf{r}^{\prime}\right|} d V
$$

The integral $I\left(\mathbf{r}^{\prime \prime}, \mathbf{r}^{\prime}\right)$ can be readily evaluated. The points $\mathbf{r}=\mathbf{r}^{\prime}$ and $\mathbf{r}=\mathbf{r}^{\prime \prime}$ are first enclosed by small spheres of volume $\epsilon$, and Green's theorem is then applied to the volume of integration $\tau-\epsilon$ outside of these spheres. We let $\tau$ be infinite. Thus,

$$
\begin{aligned}
I\left(\mathbf{r}^{\prime \prime}, \mathbf{r}^{\prime}\right)= & \frac{1}{4 \pi} \lim _{\boldsymbol{r} \rightarrow 0} \int_{\tau-\epsilon} \nabla_{r} \frac{1}{\left|\mathbf{r}-\mathbf{r}^{\prime \prime}\right|} \cdot \nabla_{r} \frac{1}{\left|\mathbf{r}-\mathbf{r}^{\prime}\right|} d V \\
= & \frac{1}{4 \pi} \lim _{\epsilon \rightarrow 0}\left\{\int \frac{1}{\left|\mathbf{r}-\mathbf{r}^{\prime \prime}\right|} \nabla_{r} \frac{1}{\left|\mathbf{r}-\mathbf{r}^{\prime}\right|} \cdot \mathbf{d S}\right. \\
& \left.-\int_{\tau \rightarrow \epsilon} \frac{1}{\left|\mathbf{r}-\mathbf{r}^{\prime \prime}\right|} \nabla^{2} \frac{1}{\left|\mathbf{r}-\mathbf{r}^{\prime}\right|} d V\right\} .
\end{aligned}
$$

The second integral is zero in the volume $\tau-\epsilon$ since $\nabla^{2}\left(1 /\left|\mathbf{r}-\mathbf{r}^{\prime}\right|\right)$ equals zero there. The first integral is evaluated over every surface present. There is no contribution from the various physical interfaces since the integrand is continuous on each of these and its value on one side of an interface thereby cancels its value on the other side during the surface integration. It also vanishes on the surface at infinity. On the surface of a small sphere about $\mathbf{r}=\mathbf{r}^{\prime \prime}$ it is readily shown that its value tends to zero as $\epsilon$ tends to zero. On the surface of a small sphere about $\mathbf{r}=\mathbf{r}^{\prime}$, its value can readily be shown to tend to $4 \pi /\left|\mathbf{r}^{\prime \prime}-\mathbf{r}^{\prime}\right|$ as $\epsilon$ tends to zero. We may conclude then that

$$
I\left(\mathbf{r}^{\prime \prime}, \mathbf{r}^{\prime}\right)=1 /\left|\mathbf{r}^{\prime \prime}-\mathbf{r}^{\prime}\right| \text {. }
$$

Introducing this into Eq. (62) it is seen that that equation is the same as the left-hand side of Eq. (56). The latter equation has therefore been established.

$$
\begin{gathered}
\text { APPENDIX II. TO SHOW THAT } \\
\int \boldsymbol{P}^{0} \cdot\left(E-E_{c}\right) d V=S P \cdot\left(E^{0}-E_{c}\right) d V(23)
\end{gathered}
$$

Taking the gradient of Eq. (2) for $\psi$ and using Eq. (19) for $\mathbf{E}_{c}$ we obtain

$$
\mathbf{E}\left(\mathbf{r}^{\prime}\right)-\mathbf{E}_{c}\left(\mathbf{r}^{\prime}\right)=-\nabla_{r^{\prime}} \int \mathbf{P}(\mathbf{r}) \cdot \nabla_{r} \frac{1}{\left|\mathbf{r}-\mathbf{r}^{\prime}\right|} d V
$$

This is actually the contribution of the polarization to the field strength, E. Similarly,

$$
\mathbf{E}^{0}\left(\mathbf{r}^{\prime}\right)-\mathbf{E}_{c}{ }^{0}\left(\mathbf{r}^{\prime}\right)=-\nabla_{r^{\prime}} \int \mathbf{P}^{0}(\mathbf{r}) \cdot \nabla_{r} \frac{1}{\left|\mathbf{r}-\mathbf{r}^{\prime}\right|} d V
$$


Introducing Eq. (66) for $\mathbf{E}-\mathbf{E}_{c}$ into the left-hand side of Eq. (23), we obtain, using in the latter equation the integration variable $\mathbf{r}^{\prime}$ instead of $\mathbf{r}$,

lhs of Eq. (23) $=-\int \mathbf{P}^{0}\left(\mathbf{r}^{\prime}\right) \cdot \nabla_{r^{\prime}}$

$$
\times\left\{\int \mathbf{P}(\mathbf{r}) \cdot \nabla_{r} \frac{1}{\left|\mathbf{r}-\mathbf{r}^{\prime}\right|} d V\right\} d V^{\prime} .
$$

Interchanging the order of integration, bringing $\mathbf{P}^{\prime}\left(\mathbf{r}^{\prime}\right) \cdot \nabla_{r^{\prime}}$ into the integral over $V$, this becomes

$$
-\int \mathbf{P}(\mathbf{r}) \cdot \nabla_{r}\left\{\int \mathbf{P}^{0}\left(\mathbf{r}^{\prime}\right) \cdot \nabla_{r^{\prime}} \frac{1}{\left|\mathbf{r}-\mathbf{r}^{\prime}\right|} d V^{\prime}\right\} d V .
$$

According to Eq. $(67)$ this is simply $\int \mathbf{P}(\mathbf{r}) \cdot\left\{\mathbf{E}^{0}(\mathbf{r})\right.$ $\left.-\mathrm{E}_{c}{ }^{0}(\mathbf{r})\right\} d V$, the right-hand side of Eq. (23).

APPENDIX III. TO SHOW THAT $\int P \cdot \mathbf{\delta}\left(E-E_{c}\right) d V=\mathcal{S}\left(E-E_{c}\right) \cdot \delta P d V(38)$

Taking the variation of Eq. (66) it is seen that the left-hand side of Eq. (38) is, using the integration variable $\mathbf{r}^{\prime}$ instead of $\mathbf{r}$ in the latter equation,

$$
-\int \mathbf{P}\left(\mathbf{r}^{\prime}\right) \cdot \nabla_{r^{\prime}}\left\{\int \delta \mathbf{P}(\mathbf{r}) \cdot \nabla_{r} \frac{1}{\left|\mathbf{r}-\mathbf{r}^{\prime}\right|} d V\right\} d V^{\prime} .
$$

Interchanging the order of integration, bringing $\mathbf{P}\left(\mathbf{r}^{\prime}\right) \cdot \nabla_{r^{\prime}}$ into the integral over $V$, this term becomes

$$
-\int \delta \mathbf{P}(\mathbf{r}) \cdot \nabla_{r}\left\{\int \mathbf{P}\left(\mathbf{r}^{\prime}\right) \cdot \nabla_{r^{\prime}} \frac{1}{\left|\mathbf{r}-\mathbf{r}^{\prime}\right|} d V^{\prime}\right\} d V .
$$

With the aid of Eq. (66) this is seen to be $\int \delta \mathbf{P}$ $\cdot\left(\mathbf{E}-\mathbf{E}_{c}\right) d V$, the right-hand side of Eq. (38).

\section{APPENDIX IV. ALTERNATIVE DERIVATION OF THE FREE ENERGY EXPRESSION, EQ. (25)}

A more intuitive but less rigorous derivation of Eq. (25) for the free energy will be presented in this Appendix.

The free energy of interaction of a dipole, whose moment is $\boldsymbol{u}$, with an electric field of strength $\mathbf{E}$ is ${ }^{13}$

$$
-\mathbf{u} \cdot \mathbf{E} \text {. }
$$

If this dipole is an induced dipole, the work required to produce it is ${ }^{12}$

$$
-\int_{\mathbf{E}=0}^{\mathbf{E}=\mathbf{E}} \boldsymbol{u} \cdot d \mathbf{E}=-\boldsymbol{u}^{2} / 2 \alpha_{0},
$$

where $\alpha_{0}$, the polarizability, is given by $\boldsymbol{u}=\alpha_{0} \mathbf{E}$. If the electric field is now turned off but the dipole moment is held fixed then there will remain a certain free energy $F_{i}$, stored up in the dipole, given by the dif-

\footnotetext{
${ }^{13}$ Reference 2, pp. 272-273.
}

ference between Eqs. (72) and (73).

$$
F_{i}=\mathbf{u}^{2} / 2 \alpha_{0} \text {. }
$$

Consider now a system consisting of a distribution of charges in a polarized medium. As before, the polarization will be composed of two types whose contributions are $\mathbf{P}_{u}(\mathbf{r})$ and $\mathbf{P}_{e}(\mathbf{r})$. The free energy of the system as a whole may be regarded as the sum of the free energies of each volume element, considered isolated, and the free energy of interaction of all the volume elements. As previously mentioned, ${ }^{2}$ the polarization can be treated as a dipole moment per unit volume. Therefore, the free energy stored up per unit volume in one of these isolated volume elements due to the production of $U$-type and $E$-type polarization is

$$
\left(\mathbf{P}_{u} \cdot \mathbf{P}_{u} / 2 \alpha_{u}\right)+\left(\mathbf{P}_{e} \cdot \mathbf{P}_{e} / 2 \alpha_{e}\right) .
$$

This follows from Eq. (74) by successively substituting there the relations $\boldsymbol{u}=\mathbf{P}_{u} d V$ and $\boldsymbol{u}=\mathbf{P}_{e} d V$ and, respectively, $\alpha_{0}=\alpha_{u} d V$ and $\alpha_{0}=\alpha_{e} d V$. The relations between the $\alpha$ 's follow from their definitions. For example, we have $\alpha_{0}=\mathbf{u} / \mathbf{E}=\mathbf{P}_{e} d V / \mathbf{E}=\left(\mathbf{P}_{e} / \mathbf{E}\right) d V=\alpha_{e} d V$.

The other contributions to the free energy of the system as a whole arise from various interactions. For example, the free energy of interaction of the $E$-type polarization of a volume element $d V$ with the field arising from the $U$-type polarization of the entire system (field strength $\mathbf{E}_{u}$, say), with that arising from all the charges (strength $\mathbf{E}_{c}$ ), and finally with that arising from the $E$-type polarization of the entire system (strength $\mathbf{E}_{\ell}$, say) is seen from $\mathbf{E q}$. (72) to be

$$
-\mathbf{P}_{e} \cdot\left(\mathbf{E}_{u}+\mathbf{E}_{c}+\mathbf{E}_{\boldsymbol{e}}\right) d V \text {. }
$$

Similarly, the free energy of interaction of the $U$-type polarization of the volume element with the fields $\mathbf{E}_{u}, \mathbf{E}_{c}$, and $\mathbf{E}_{\varepsilon}$ is

$$
-\mathbf{P}_{u} \cdot\left(\mathbf{E}_{u}+\mathbf{E}_{c}+\mathbf{E}_{\varepsilon}\right) d V .
$$

Finally, there is the free energy of interaction of the charges with each other. This term is the same as the corresponding quantity for charges in a vacuum, since the terms describing the interaction of the charges with the polarization have already been considered. An examination of the usual expression ${ }^{14}$ for the interaction of charges in a vacuum, and the definition of $\mathbf{E}_{c}$ given in Eq. (19), shows that this term is, per unit volume,

$$
\mathbf{E}_{c} \cdot \mathbf{E}_{c} / 8 \pi
$$

The expression for the electrostatic free energy of the system is then obtained by summing all these terms and integrating over the entire volume of the system. In performing the integration several of these terms should be divided by a factor of two; otherwise, certain interactions would be counted twice. These terms are

$$
-\int\left(\mathbf{P}_{e} \cdot \mathbf{E}_{e}+\mathbf{P}_{u} \cdot \mathbf{E}_{u}+\mathbf{P}_{e} \cdot \mathbf{E}_{u}+\mathbf{P}_{u} \cdot \mathbf{E}_{e}\right) d V .
$$

${ }^{14}$ Reference 2, p. 275. 
We thus obtain

$$
\begin{array}{r}
F=\int\left\{\frac{\mathbf{E}_{c}^{2}}{8 \pi}+\frac{\mathbf{P}_{u}^{2}}{2 \alpha_{u}}+\frac{\mathbf{P}_{e}^{2}}{2 \alpha_{e}}-\mathbf{P}_{e} \cdot\left(\mathbf{E}_{c}+\frac{\mathbf{E}_{u}}{2}+\frac{\mathbf{E}_{e}}{2}\right)\right. \\
\left.-\mathbf{P}_{u} \cdot\left(\mathbf{E}_{c}+\frac{\mathbf{E}_{u}}{2}+\frac{\mathbf{E}_{e}}{2}\right)\right\} d V .
\end{array}
$$

The electric field strength $\mathbf{E}$ is simply the sum $\left(\mathbf{E}_{\iota}+\mathbf{E}_{u}+\mathbf{E}_{\boldsymbol{e}}\right)$. Introducing this and the relations, $\mathbf{P}_{e}=\alpha_{e} \mathbf{E}, \mathbf{P}=\mathbf{P}_{e}+\mathbf{P}_{u}$, into Eq. (80) we obtain

$$
F=\int\left\{\frac{\mathbf{E}_{c}^{2}}{8 \pi}+\frac{\mathbf{P}_{u}{ }^{2}}{2 \alpha_{u}}-\frac{\mathbf{P} \cdot \mathbf{E}_{c}}{2}-\frac{\mathbf{P}_{u} \cdot \mathbf{E}}{2}\right\} d V
$$

which is identical with Eq. (25).

THE JOURNAL OF CHEMICAL PHYSICS

VOLUME 24, NUMBER 5

$\mathrm{MAY}, 1956$

\title{
Normal Vibration Frequencies of $\mathrm{CD}_{3} \mathrm{~F}$. Structure of $\mathrm{CH}_{3} \mathrm{~F}$ and $\mathrm{CD}_{3} \mathrm{~F}$ from Infrared and Microwave Spectra
}

\author{
F. Allan Andersen, B $\emptyset$ Rge Bak, and Svend Brodersen \\ Chemical Laboratory of the University of Copenhagen, Copenhagen, Denmark
}

(Received July 22, 1955)

\begin{abstract}
The infrared absorption spectrum of $\mathrm{CD}_{3} \mathrm{~F}$ has been studied. Simultaneously, the infrared spectrum of $\mathrm{CH}_{3} \mathrm{~F}$ was reinvestigated. Fundamental vibration frequencies for $\mathrm{CD}_{3} \mathrm{~F}$ were found together with Coriolis coupling factors for the three degenerate vibrations in $\mathrm{CH}_{3} \mathrm{~F}$ and $\mathrm{CD}_{3} \mathrm{~F}$. The fine-structure analysis gave reliable values for the large rotational constants of $\mathrm{CD}_{3} \mathrm{~F}$ and $\mathrm{CH}_{3} \mathrm{~F}$. The structure of the methyl fluoride molecule is discussed.
\end{abstract}

\section{INTRODUCTION}

A $T$ present, fundamental vibration frequencies for the three pairs, $\mathrm{CH}_{3} \mathrm{X}, \mathrm{CD}_{3} \mathrm{X}$ where $\mathrm{X}=\mathrm{Cl}, \mathrm{Br}$, and $I$ have been reported in the literature ${ }^{1}$ together with data for $\mathrm{CH}_{3} \mathrm{~F}^{2-4}$ Fundamental vibration frequencies for $\mathrm{CD}_{3} \mathrm{~F}$ represent, therefore, a very desirable supplement. In an attempt to produce these data, $\mathrm{CD}_{3} \mathrm{~F}$ was prepared and its infrared absorption was measured. Also, the spectrum of $\mathrm{CH}_{3} \mathrm{~F}$ was reinvestigated. Values of the frequencies of the parallel vibrations followed by direct inspection while the localization of the centers of the perpendicular bands necessitated a thorough analysis of the fine structure. Simultaneously, these analyses resulted in values for the small moment of inertia of $\mathrm{CH}_{3} \mathrm{~F}$ and $\mathrm{CD}_{3} \mathrm{~F}$ and the Coriolis coupling factors for both molecular species.

\section{EXPERIMENTAL PART}

\section{A. Preparations}

$\mathrm{CH}_{3} \mathrm{~F}$ and $\mathrm{CD}_{3} \mathrm{~F}$ were prepared from $\mathrm{CH}_{3} \mathrm{Cl}$ and $\mathrm{CD}_{3} \mathrm{Cl}$ (synthesized by the method of Noether ${ }^{5}$ ) and high-grade AgF. ${ }^{6}$ The state of purity of the two methyl chlorides was checked by control of their infrared absorption. In addition, a mass spectrometer run on $\mathrm{CD}_{3} \mathrm{Cl}$ showed the purity of the sample to be well over

\footnotetext{
${ }^{1}$ G. Herzberg, Infrared and Raman Spectra (D. Van Nostrand Company, Inc., New York, 1945), p. 315.

2 W. H. Bennett, and C. F. Meyer, Phys. Rev. 32, 888 (1928).

${ }^{3}$ K. P. Yates, and H. H. Nielsen, Phys. Rev. 71, 349 (1947).

${ }^{4}$ J. Pickworth, and H. W. Thompson, Proc. Roy. Soc. (London) A222, 443 (1954)

${ }^{5}$ H. D. Noether, J. Chem. Phys. 10, 664 (1942).

Andersen, Bak, and Hillebert, Acta Chem. Scand. 7, 236 (1953).
}

$90 \%$. -0.0057 mole $\mathrm{CH}_{3} \mathrm{Cl}$ (or $\mathrm{CD}_{3} \mathrm{Cl}$ ) was kept heated with 0.032 mole $\mathrm{AgF}$ (4.0 g) for 4 hours in a $250-\mathrm{ml}$ sealed-off glass container at $225^{\circ} \mathrm{C}$. After cooling, an infrared spectrum of the gaseous reaction mixture was taken which showed that about $20 \%$ of the methyl chloride (I) had not reacted. Furthermore, a small amount of a nonidentified compound (II) was detected together with a surprisingly large amount of carbon dioxide (probably originating from a reaction between the glass, the silver fluoride, and the organic compounds). The reaction mixture was analyzed on a 50-cm dry silica-gel column (internal diameter : $4 \mathrm{~mm}$ ) and $\mathrm{Pt}$-wire detector at room temperature. Peaks corresponding to $\mathrm{CO}_{2}$, methyl fluoride, and (I) were clearly visible. At a subsequent separation run (I) and (II) were removed quantitatively as demonstrated by the infrared spectrum. In a second separation run through the column all the $\mathrm{CO}_{2}$ and part of the methyl fluoride was removed, which produced a final ("light" or "heavy") methyl fluoride completely free of $\mathrm{CO}_{2}$. In spite of the somewhat wasteful separation procedure the final yield of highly purified product was $35 \%$.

\section{B. Spectroscopic Procedure and the Spectra}

The spectra were taken on a Beckman IR 3 instrument with suitable optics $(\mathrm{NaCl}$ and $\mathrm{LiF})$. The effective slit width and the pressure applied will be given at the top of the tables containing the experimental results. The cell length was constantly $10 \mathrm{~cm}$. The absolute magnitude of our frequencies may deviate about $\frac{1}{2} \mathrm{~cm}^{-1}$ from the true values but apart from this or other kinds of systematic errors (such as those originating from our limited resolving power) it is 